OPEN ACCESS

Edited by:

Mariano Martinez-Vazquez,

National Autonomous University

of Mexico, Mexico

Reviewed by:

Phil Rather

Emory University, United States

Joseph Boll,

University of Texas at Arlington,

United States

Jintae Lee,

Yeungnam University, South Korea

*Correspondence:

Ana Otero

anamaria.otero@usc.es

${ }^{\dagger}$ These authors have contributed equally to this work

Specialty section:

This article was submitted to

Antimicrobials, Resistance

and Chemotherapy,

a section of the journal

Frontiers in Microbiology

Received: 25 May 2020 Accepted: 10 September 2020 Published: 30 September 2020

Citation:

Mayer C, Muras A, Parga A, Romero M, Rumbo-Feal S, Poza M, Ramos-Vivas J and Otero A (2020)

Quorum Sensing as a Target for Controlling Surface Associated

Motility and Biofilm Formation in Acinetobacter baumannii ATCC ${ }^{\circledR}$ $17978^{\text {TM }}$

Front. Microbiol. 11:565548. doi: 10.3389/fmicb.2020.565548

\title{
Quorum Sensing as a Target for Controlling Surface Associated Motility and Biofilm Formation in Acinetobacter baumannii ATCC ${ }^{\circledR}$ $17978^{\mathrm{TM}}$
}

Celia Mayer $1,2+$, Andrea Muras ${ }^{1 \dagger}$, Ana Parga $^{1}$, Manuel Romero $^{2}$, Soraya Rumbo-Feal
Margarita Poza $^{3}$, José Ramos-Vivas

${ }^{1}$ Departamento de Microbioloxía e Parasitoloxía, Facultade de Bioloxía, Edificio CIBUS, Universidade de Santiago de Compostela, Santiago de Compostela, Spain, ${ }^{2}$ National Biofilms Innovation Centre, Biodiscovery Institute and School of Life Sciences, University of Nottingham, Nottingham, United Kingdom, ${ }^{3}$ Microbioloxía, Instituto de Investigación Biomédica da Coruña, Centro de Investigacións Cientificas Avanzadas da Coruña, Universidade da Coruña, A Coruña, Spain, ${ }^{4}$ Servicio de Microbiología, Hospital Universitario Marqués de Valdecilla-Instituto de Investigación Valdecilla, Santander, Spain

The important nosocomial pathogen Acinetobacter baumannii presents a quorum sensing (QS) system (abal/abaR) mediated by acyl-homoserine-lactones (AHLs) and several quorum quenching $(\mathrm{QQ})$ enzymes. However, the roles of this complex network in the control of the expression of important virulence-related phenotypes such as surface-associated motility and biofilm formation is not clear. Therefore, the effect of the mutation of the AHL synthase Abal, and the exogenous addition of the $Q Q$ enzyme Aii20J on surface-associated motility and biofilm formation by $A$. baumannii ATCC ${ }^{\oplus} 17978^{\mathrm{TM}}$ was studied in detail. The effect of the enzyme on biofilm formation by several multidrug-resistant $A$. baumannii clinical isolates differing in their motility pattern was also tested. We provide evidence that a functional QS system is required for surface-associated motility and robust biofilm formation in A. baumannii ATCC ${ }^{\circledR}$ $17978^{\mathrm{TM}}$. Important differences were found with the well-studied strain A. nosocomialis M2 regarding the relevance of the QS system depending on environmental conditions The in vitro biofilm-formation capacity of $A$. baumannii clinical strains was highly variable and was not related to the antibiotic resistance or surface-associated motility profiles. A high variability was also found in the sensitivity of the clinical strains to the action of the $Q Q$ enzyme, revealing important differences in virulence regulation between A. baumannii isolates and confirming that studies restricted to a single strain are not representative for the development of novel antimicrobial strategies. Extracellular DNA emerges as a key component of the extracellular matrix in A. baumannii biofilms since the combined action of the QQ enzyme Aii20J and DNase reduced biofilm formation in all tested strains. Results demonstrate that $Q Q$ strategies in combination with other enzymatic treatments such as DNase could represent an alternative approach for the prevention of $A$. baumannii colonization and survival on surfaces and the prevention and treatment of infections caused by this pathogen.

Keywords: Acinetobacter baumannii, quorum sensing, surface-associated motility, biofilm, quorum quenching, extracellular DNA 


\section{INTRODUCTION}

The genus Acinetobacter comprises Gram-negative, strictly aerobic coccobacilli that are widely present in the environment but also includes a variety of species that cause opportunistic nosocomial infections like septicemia, pneumonia, endocarditis, meningitis, skin, wound, and urinary tract infections (Towner, 2009). Acinetobacter baumannii, the most relevant pathogenic species in the genus, has emerged as one of the most troublesome hospital-acquired pathogens because the increase in the prevalence of multidrug-resistant (MDR) strains has reduced the treatment options for this pathogen (Peleg et al., 2008; Roca et al., 2012; Lee et al., 2017; Moubareck and Halat, 2020). Therefore, a better understanding of the mechanisms controlling the expression of virulence traits and propagation in Acinetobacter spp. has become critical for the discovery and development of new therapeutic strategies.

In Acinetobacter spp. a complex network of sensors controls the expression of virulence factors integrating intra- and extracellular signals (Harding et al., 2018; Wood et al., 2018a; De Silva and Kumar, 2019). Among these, quorum sensing (QS), a mechanism of control of gene expression dependent on bacterial cell density, seems to play a central role, constituting an interesting target for the development on antimicrobial strategies. Acinetobacter spp. present a QS system homologous to the canonical LuxI/LuxR system of Gram-negatives that produces and sense QS signals belonging to the family of the acylhomoserine-lactones (AHLs) that is known as AbaI/AbaR (Niu et al., 2008; Bhargava et al., 2010; Mayer et al., 2018). The system was first identified in A. nosocomialis M2, formerly classified as A. baumannii (Carruthers et al., 2013) and later found in A. baumannii (Smith et al., 2007; Niu et al., 2008; Mayer et al., 2018) and other species of the genus of both human or environmental origin (Kang and Park, 2010; Bitrian et al., 2012; How et al., 2015; Oh and Choi, 2015). N-hydroxydodecanoylL-homoserine lactone (OHC12-HSL) is the main QS signal produced by the clinically relevant species belonging to the A. calcoaceticus-A. baumannii complex, including the wellstudied strains $A$. nosocomialis $\mathrm{M} 2$ and $A$. baumannii ATCC $^{\circledR}$ $17978^{\mathrm{TM}}$, although other AHLs are also produced in smaller amounts (Niu et al., 2008; Chan et al., 2011, 2014; Clemmer et al., 2011; How et al., 2015; Mayer et al., 2018). In vitro, these signals are produced only in static cultures (Mayer et al., 2018), indicating a strong correlation between QS and biofilm formation and/or surface attachment. Besides producing QS signals, the capacity to enzymatically degrade these molecules, a process known as Quorum Quenching (QQ), has been described in several Acinetobacter spp. of environmental and clinical origin (Kang et al., 2004; Chan et al., 2011; Kim et al., 2014; Ochiai et al., 2014; Arivett et al., 2015; López et al., 2017; Mayer et al., 2018). Up to 8 putative QQ enzymes have been found in the genome of A. baumannii ATCC ${ }^{\circledR} 17978^{\mathrm{TM}}$ and, in this strain, QQ activity correlates with the disappearance of the AHLs from the culture media, indicating that AHL production may be self-regulated (Mayer et al., 2018).

QS controls many different functions in Acinetobacter spp., including some key virulence factors such as motility, biofilm formation and other stress responses (Gaddy and Actis, 2009; Roca et al., 2012; Eze et al., 2018; Harding et al., 2018; Mayer et al., 2020; Shin et al., 2020), although most work was done with A. nosocomialis M2 (Niu et al., 2008; Clemmer et al., 2011; Stacy et al., 2012; Oh and Choi, 2015). Surfaceassociated motility is related to virulence since it has been identified as a common trait in clinical isolates of $A$. baumannii (Eijkelkamp et al., 2011) and increased motility has been related to increased adherence and lethality in Caenorhabditis elegans assays (Eijkelkamp et al., 2013). Several regulatory proteins have been described as involved in the control of surface-associated motility in Acinetobacter spp. (Mayer et al., 2018; Moubareck and Halat, 2020). The AHL-mediated QS system seems to be central in this regulatory network since an impairment in surface motility was recorded for QS defective mutants in A. nosocomialis, a phenotype that could be restored by the addition of OHC12-HSL (Clemmer et al., 2011; Oh and Choi, 2015). The role of QS on motility in A. baumannii is less studied, but recently, important differences in motility characteristics have been reported for clinical isolates of A. baumannii differing in their antibiotic resistance (López et al., 2017). In A. baumannii ATCC $^{\circledR} 17978^{\mathrm{TM}}$ (Mayer et al., 2018) and in some but not all the clinical strains studied (López et al., 2017), the addition of the AHL-degrading enzyme Aii20J reduced or even blocked motility completely, supporting a role of AHL-mediated QS on this phenotype in A. baumannii, as previously described for A. nosocomialis. However, the effect of the mutation of the QS genes on motility in A. baumannii and its correlation with biofilm formation has not been studied so far.

Acinetobacter baumannii has a strong ability to form biofilms, a capacity that has been linked to this species resistance to desiccation, nutrient starvation, and antimicrobial treatments (Gaddy and Actis, 2009). Potential virulence genes, including those involved in antibiotic resistance, seem to be overexpressed in A. baumannii biofilms (Marti et al., 2011; Rumbo-Feal et al., 2017) and a positive correlation was found between biofilm formation and multiple drug resistance in A. baumannii clinical isolates (Yang et al., 2019; Zeighami et al., 2019). The infective capacity and the development of chronic infections are related to the capacity to form biofilms (Eze et al., 2018) since genes required for biofilm formation are widespread in A. baumannii clinical strains but are partially or completely missing in environmental isolates (Yakkala et al., 2019). Numerous evidence correlates surface-associated motility and biofilm formation in several species and strains of the genus Acinetobacter (Stacy et al., 2012; Tucker et al., 2014; Giles et al., 2015; Chen et al., 2017), indirectly indicating that both traits are controlled by QS (Niu et al., 2008; Gaddy and Actis, 2009; Bhargava et al., 2010; Anbazhagan et al., 2012; Stacy et al., 2012; Chen et al., 2017; Rumbo-Feal et al., 2017). A hyper-motile variant of A. baumannii ATCC $^{\circledR} 17978^{\mathrm{TM}}$ lost its motility and produced less pellicle when two genes of the QS genomic region were mutated (Giles et al., 2015). The reduction of biofilm formation by QQ lactonases has been already demonstrated in clinical isolates of A. baumannii (Chow et al., 2014; Zhang et al., 2017) although the effect of these enzymes on biofilm formation was low in comparison with a QS null mutant (Chow et al., 2014). The exogenous 
addition of the AHL-lactonase Aii20J strongly inhibited biofilm formation in A. baumannii ATCC ${ }^{\circledR} 17978^{\mathrm{TM}}$ despite the presence of endogenous QQ activity (Mayer et al., 2018), indicating that the endogenous enzymes might be involved in the fine-tuning of the QS system and/or be active during specific periods or conditions. On the contrary, a previous study showed that the AHL-lactonase MomL showed little or no-activity activity on A. nosocomialis M2 and several clinical isolates and lost its antibiofilm activity when a QQ-sensitive A. baumannii strain was co-cultured with Pseudomonas aeruginosa PAO1 (Zhang et al., 2017). It should be noted that most biofilm assays are performed in microtiter plates in which the formation of biofilm by this strictly aerobic species is weak and highly variable. Therefore, to assess the potential use of QQ enzymes for the control of these important virulence traits in A. baumannii, it is necessary to assess the effect of the inactivation of $a b a I$ and the addition of QQ enzymes in conditions that drive to robust biofilm formation and to evaluate the response of different clinical isolates in comparison with A. baumannii ATCC ${ }^{\circledR} 17978^{\mathrm{TM}}$. Moreover, extracellular DNA (eDNA) is present in A. baumannii biofilms and a reduction of biofilm formation by DNase I has been already reported, even in preformed biofilms (Tetz et al., 2009; Sahu et al., 2012) and therefore, the impact of combining QQ strategies with eDNA digestion deserves further exploration.

The key role of QS in the control of virulence factors, including surface-associated motility and biofilm formation in different species/strains of Acinetobacter points to the use of QQ enzymes as a possible antimicrobial strategy in these species. Nevertheless, previous studies revealed important differences between species and strains (Vijayakumar et al., 2016; López et al., 2017; Zhang et al., 2017). Therefore, a more detailed study of the role of QS in surface-associated and biofilm formation in A. baumannii ATCC $^{\circledR} 17978^{\mathrm{TM}}$ and a comparison with clinical strains is required. In order to better understand the influence of QS regulatory mechanisms on these virulence-associated traits in the reference strain A. baumannii ATCC ${ }^{\circledR} 17978^{\mathrm{TM}}$, in this work, we used a QS defective mutant and QQ strategies. Data presented here reveal that QS regulates both traits in A. baumannii ATCC $^{\circledR}$ $17978^{\mathrm{TM}}$ and its behavior differs from A. nosocomialis M2. The study of biofilm formation capacity of different multidrugresistant A. baumannii strains and their susceptibility to the QQ enzyme Aii20J and other biofilm-disrupting enzymes revealed a great variability among clinical isolates, indicating that studies targeting the development of novel antimicrobial strategies should be based on multiple strains of different origin and characteristics. Extracellular DNA emerges as a key component of extracellular matrix in Acinetobacter biofilms, since the combined action of the QQ enzyme Aii20J and a DNase reduced biofilm formation in all the tested strains.

\section{MATERIALS AND METHODS}

\section{Bacterial Strains, Culture Conditions, and Genetic Methods}

Bacterial strains and primers used in this study are listed in Table 1. Luria-Bertani (LB) broth and LB agar were used to grow and maintain Acinetobacter spp. routinely at $37^{\circ} \mathrm{C}$. LB broth was prepared in our laboratory with $1 \%$ Bacto-tryptone (Life Technologies Corporation, Thermo Fisher Scientific), 0.5\% yeast extract (BD Biosciences) and 1\% NaCl (Panreac AppliChem).

\section{Construction of Isogenic Deletion Derivatives}

Plasmid pMo130, a suicide vector containing the genes $x y l E$, $s a c B$ and a kanamycin resistance marker, was used as described by Hamad et al. (2009). Briefly, 900-1000 bp upstream and downstream regions flanking the genes selected for deletion in A. baumannii ATCC ${ }^{\circledR} 17978^{\mathrm{TM}}$ were PCR-amplified and cloned into the pMo130 vector using primers listed in Table 1. The resulting plasmid (pMo130-0109) and (pMo130-1750) were transformed into ATCC ${ }^{\circledR} 17978^{\mathrm{TM}}$ cells by electroporation (Rumbo-Feal et al., 2013). Recombinant colonies representing the first crossover event were selected by resistance to kanamycin and visual detection of XylE activity following the catechol-based method (Hamad et al., 2009). Bright yellow kanamycin-resistant colonies were then grown overnight in LB supplemented with $15 \%$ sucrose and then plated on LB agar without antibiotics. Second crossover event leading to gene deletion was then confirmed by PCR using primers listed in Table 1. The $\triangle 0109$ isogenic deletion and derivative of ATCC ${ }^{\circledR}$ $17978^{\mathrm{TM}}$ was constructed by deleting a region encompassing the A1S_0109 gene, without affecting the upstream and downstream surrounding genes as described previously (Hamad et al., 2009).

\section{Motility Assays}

Surface-associated motility assays were performed as described by Mayer et al. (2018). Petri dishes were prepared with LB or low-nutrients low-salt $\mathrm{LB}(0.5 \% \mathrm{NaCl}, 0.2 \%$ tryptone, and $0.1 \%$ yeast extract; LNLS-LB) media supplemented with $0.25 \%$ Difco (Bacto ${ }^{\text {TM }}$ Agar) or Eiken agar (Eiken Chemical, Co. Ltd., Japan). Plates with a reduced concentration of $\mathrm{NaCl}$ $(0.5 \%)$, tryptone $(0.2 \%)$, or yeast extract $(0.1 \%)$ or media with different concentrations of $\mathrm{NaCl}(0.1-0.4 \mathrm{M})$ or sucrose (5-20\%) were also prepared to determinate the impact of culture media or osmolarity effect on motility, respectively. $\mathrm{N}$-Hydroxydodecanoyl-L-homoserine lactone (OHC12-HSL from Sigma, $10 \mu \mathrm{M})$, or the AHL-lactonase Aii20J $(20 \mu \mathrm{g} / \mathrm{mL})$ obtained from the marine bacterium Tenacibaculum sp. 20J (Mayer et al., 2015) were mixed with the inoculum or with the culture media. One microliter from agitated overnight cultures at 0.3 optical density $\left(\mathrm{OD}_{600} \mathrm{~nm}\right)$ was inoculated in the center of the plates. Plates were incubated at $37^{\circ} \mathrm{C}$ in the dark, and surface-associated motility was inspected after $14 \mathrm{~h}$. Three plates were prepared for each condition, and experiments were repeated at least twice.

\section{Assessment of Biofilm Formation by Acinetobacter spp.}

Biofilms were grown in a modification of the Amsterdam Active Attachment model (AAA-model) (Exterkate et al., 2010; Muras et al., 2020) assembled with glass coverslips (18 $\mathrm{mm} \times 18 \mathrm{~mm})$. In 
TABLE 1 | Bacterial strains, and primers used in this study.

\begin{tabular}{|c|c|c|}
\hline Strains & Description & Source or reference \\
\hline \multicolumn{3}{|l|}{ Acinetobacter baumannii } \\
\hline ATCC ${ }^{\circledR} 17978^{\mathrm{TM}}$ & & ATCC $^{a}$ \\
\hline$\Delta a b a l\left(\right.$ ATCC $\left.^{\circledR} 17978^{\mathrm{TM}}\right)$ & د0109 knock out (KO) mutant without synthase AHLs gene abal (A1S_0109) & This study \\
\hline Ab1 (ROC013) & A. baumannii clinical isolate (respiratory). TM: ST2 & López et al., 2017 \\
\hline Ab4 (VAL001) & A. baumannii clinical isolate (respiratory). TM: ST169 & López et al., 2017 \\
\hline Ab5 (DOM009) & A. baumannii clinical isolate (respiratory). TM: ST80 & López et al., 2017 \\
\hline Ab7 (HUI001) & A. baumannii clinical isolate (respiratory). TM: ST79 & López et al., 2017 \\
\hline MAR002 & A. baumannii biofilm hyper-producing clinical isolate (wound sample) TM: ST271 & Álvarez-Fraga et al., 2016 \\
\hline$\Delta 11085$ & MAR002 mutant defective in biofilm formation and cell attachment & Álvarez-Fraga et al., 2016 \\
\hline \multicolumn{3}{|l|}{ Acinetobacter nosocomialis } \\
\hline M2 & & Niu et al., 2008 \\
\hline abal:Km (M2) & abal mutant (abal:Km), $\mathrm{Km}^{r}$ & Niu et al., 2008 \\
\hline \multicolumn{3}{|l|}{ Escherichia coli } \\
\hline $\begin{array}{l}\text { BL21(DE3)plysS pET28c(+)- } \\
\text { aii20J }\end{array}$ & $\begin{array}{l}\text { E. coli containing a cloning vector }\left(\mathrm{pET} 28 \mathrm{c}(+), \mathrm{Km}^{r}\right) \text { and aii20J gene from } \\
\text { Tenacibaculum sp. } 20 \mathrm{~J}\end{array}$ & Mayer et al., 2015 \\
\hline BL21(DE3)plysS pET28c(+) & E. coli containing a cloning vector without the aii20J gene & Mayer et al., 2015 \\
\hline Plasmids & Reference & Use in this work \\
\hline pMo130 & GenBank: EU862243 & Construction of knockout strains \\
\hline Primers & Sequence $\left(5^{\prime}-3^{\prime}\right)$ & Use in this work \\
\hline 0109UpFPstl & CCCCTGCAGGGGACTGGTGTCGTTATTACC & Construction of knockout strain $\Delta 0109$ \\
\hline 0109UpREcoRI & GGGGAATTCCCCCTTGGAGTAGAACGTTATTA & Construction of knockout strain $\Delta 0109$ \\
\hline 0109DownFEcoRI & CCCGAATTCGGGACATAGGCTGTATCGACTT & Construction of knockout strain $\Delta 0109$ \\
\hline 0109DownRBamHI & GGGGGATCCCCCACTGTAGAAATCCCTATACTT & Construction of knockout strain $\Delta 0109$ \\
\hline 0109extF & TGTTCCCGATTATGTATG & Confirmation of $\Delta 0109$ \\
\hline 0109extR & GCAACTTCACAAACTCCA & Confirmation of $\Delta 0109$ \\
\hline pMo130site2F & ATTCATGACCGTGCTGAC & Checking of pMo130 \\
\hline pMo130site2R & CTTGTCTGTAAGCGGATG & Checking of pMo130 \\
\hline
\end{tabular}

a American type culture collection.

short, coverslips were vertically submerged in $3 \mathrm{~mL}$ of LB or LSLB $(0.5 \% \mathrm{NaCl}, 1 \%$ tryptone, and $0.5 \%$ yeast extract) inoculated with 24 h-cultures of $A$. baumannii strains at an optical density of $0.05\left(\mathrm{OD}_{600} \mathrm{~nm}\right)$ in 12-well culture plates. Biofilms were grown at $37^{\circ} \mathrm{C}$ for 12,24 , or $48 \mathrm{~h}$. The QQ enzyme Aii20J (Mayer et al., 2015) at a final concentration of $20 \mu \mathrm{g} / \mathrm{mL}$, DNase at $2 \mathrm{U} / \mathrm{mL}$, $\alpha$-amylase $(10 \mathrm{U} / \mathrm{mL})$ were added to the cultures alone or in combination. Wells with only culture medium were incubated in the same conditions as negative growth controls. Three replicates for each strain or treatment were performed. For biofilm biomass quantification, after incubation, the coverslips were removed, deposited in a clean 12-wells culture plate, and allowed to dry in a clean bench. Once dried, wells were filled with Crystal Violet solution (0.04\%, Gram-Hucker, Panreac), and after $20 \mathrm{~min}$, the excess of dye was removed, and coverslips washed several times with distilled water. Bound crystal violet was released by adding 33\% acetic acid. The absorbance was measured at $600 \mathrm{~nm}$ (Muras et al., 2020).

\section{Transmission Electron Microscopy}

Biofilms and planktonic cells of A. baumannii ATCC ${ }^{\circledR} 17978^{\mathrm{TM}}$, its isogenic mutant $\triangle a b a I$, and A. baumannii ATCC $^{\circledR} 17978^{\mathrm{TM}}$ supplemented with $20 \mu \mathrm{g} / \mathrm{mL}$ Aii20J enzyme, were examined by transmission electron microscopy (TEM) to confirm the presence of surface appendages. Coverslips were incubated for $24 \mathrm{~h}$ in LS-LB as explained above. Biofilms and planktonic cells obtained by centrifugation $(1 \mathrm{~mL})$ were fixed with ice-cold 3.5\% paraformaldehyde for $20 \mathrm{~min}$ at $4^{\circ} \mathrm{C}$. Bacteria were applied to Formvar-coated grids and were air-dried. At least two different formvar coated grids prepared from two different experiments. The cells were then negatively stained with $1 \%$ phosphotungstic acid in distilled water for $5 \mathrm{~s}$ and were examined with a JEM-1011 transmission electron microscope (JEOL) operating at $80 \mathrm{kV}$ and equipped with an Orius SC1000 charge-coupled device (CCD) camera (Gatan).

\section{Statistical Methods}

Mann-Whitney test $(p<0.05)$ was applied for all statistical analyses using GraphPad Prisma 8.3.0.

\section{RESULTS}

\section{QS and Surface-Associated Motility in A. baumannii ATCC ${ }^{\circledR}$ 17978 $^{\mathrm{TM}}$}

The deletion of gene A1S_0109, encoding a putative AHL synthase in $A$. baumannii ATCC $^{\circledR} 17978^{\mathrm{TM}}$, showing an amino acid sequence identity of $93.4 \%$ with the AbaI 


A. baumannii ATCC17978
A. nosocomialis $\mathbf{M 2}$
FIGURE 1 I Effect of the mutation of the abal gene on surface-associated
motility of $A$. baumannii $\mathrm{ATCC}{ }^{\circledR} 17978^{\mathrm{TM}}$, and $\mathrm{A}$. nosocomialis M2. The
capacity of OHC12-HSL (10 $\mu \mathrm{M})$ to restore the phenotype in the mutants was
tested. Plates were prepared in $\mathrm{LB}$ or low-nutrient low-salt LB (LNLS-LB) with
0.25\% Eiken agar and were incubated at $37^{\circ} \mathrm{C}$ for 14 h. Images are
representative of three independent experiments carried out with three
replicates per condition.

synthase of A. nosocomialis M2 (Carruthers et al., 2013), caused the disappearance of AHLs from the culture media under the conditions that promoted the production of these signals in the wild-type (Mayer et al., 2018). No differences in growth were observed between the $\Delta a b a I$ mutant and the parental strain in shaken liquid cultures (data not shown).

LB medium completely hindered surface-associated migration in A. baumannii ATCC ${ }^{\circledR} 17978^{\mathrm{TM}}$ (Figure 1), while a "tentaclelike," branched motility pattern was observed in LNLS-LB for this species at $37^{\circ} \mathrm{C}$. This phenotype was not affected by the type of agar used (Supplementary Figure 1). On the contrary, A. nosocomialis M2 maintained the motility in both LB and LNLS-LB (Figure 1) but only on plates prepared with Eiken agar (Supplementary Figure 1). Deletion of the abaI gene completely abolished motility under permissive conditions in A. baumannii ATCC ${ }^{\circledR} 17978^{\mathrm{TM}}$ while almost no effect could be observed in the $A$. nosocomialis M2 abaI:Km mutant at $37^{\circ} \mathrm{C}$ (Figure 1). The exogenous addition of $\mathrm{OHC12}$ HSL, the major AHL signal found in the supernatants of A. baumannii ATCC ${ }^{\circledR} 17978^{\mathrm{TM}}$ and A. nosocomialis M2 cultures (Niu et al., 2008; Mayer et al., 2018) was able to restore this phenotype in the $\triangle a b a I$ mutant of $\mathrm{ATCC}^{\circledR} 17978^{\mathrm{TM}}$ under permissive conditions, confirming that AHL-mediated QS is crucial for surface-associated motility in this species, while no significant effect was observed when added to the wild type (Figure 1).

A systematic study of plate motility was performed by reducing the concentrations of each $\mathrm{LB}$ medium component to that of LNLS-LB to define better the LB-medium component responsible for abrogating surface-associated motility in A. baumannii ATCC17978 and its effect on the role of the QS system. The experiments were performed in Eiken agar, that was shown to be more motile-permissive for A. nosocomialis M2 strain (Supplementary Figure 1). Results showed that a reduction in salt concentration from 1 to $0.5 \%$ while keeping

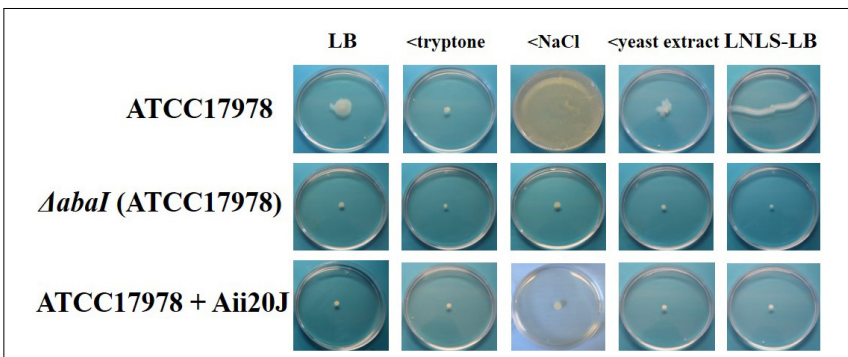

FIGURE 2 | Effect of the components of LB culture medium on surface-associated motility of $A$. baumannii ATCC ${ }^{\circledR} 17978^{\text {TM }}$ and its isogenic mutant $\triangle a b a l$, on different media plates with $0.25 \%$ Eiken agar. The effect of the QQ enzyme Aii20J $(20 \mu \mathrm{g} / \mathrm{mL})$ on A. baumannii ATCC ${ }^{\circledR} 17978^{\text {TM }}$ was also evaluated. Plates were incubated at $37^{\circ} \mathrm{C}$ for $14 \mathrm{~h}$ and images are representative of three independent experiments carried out carried out with three replicates per condition ( < tryptone: LB with reduced tryptone concentration, < $\mathrm{NaCl}$ : LB with reduced $\mathrm{NaCl}$ concentration, < yeast extract: LB with reduced yeast extract concentration, and LNLS-LB: low-nutrient low-salt LB).

other LB constituent concentrations, promotes a hyper-motile phenotype in ATCC $^{\circledR} 17978^{\mathrm{TM}}$ (Figure 2) similar to that observed in M2 in LB medium (Figure 1 and Supplementary Figure 2). Lowering tryptone or yeast extract concentrations did not change the non-motile phenotype observed in LB (Figure 2), indicating that $\mathrm{NaCl}$ concentrations higher than $0.5 \%$ are responsible for the inhibition of motility observed in LB medium for ATCC ${ }^{\circledR} 17978^{\mathrm{TM}}$. Additional experiments carried out by substituting $\mathrm{NaCl}$ by sucrose indicated that high osmolarity conditions, and not simply ionic strength, are responsible for the abrogation of motility in this strain (Supplementary Figure 3). In the case of $A$. nosocomialis $\mathrm{M} 2$, the reduction of tryptone, the main source of $\mathrm{C}$ and $\mathrm{N}$ in $\mathrm{LB}$ medium, was responsible for the change in the motility pattern observed in LNLS-LB (Figure 1), with no effect of $\mathrm{NaCl}$ concentration (Supplementary Figure 2). The loss of surface-associated motility capacity in the $\triangle a b a I$ mutant of ATCC ${ }^{\circledR} 17978^{\mathrm{TM}}$ was confirmed in all the tested media (Figure 2). The exogenous addition of the QQ enzyme Aii20J, mixed with the inoculum, had a similar effect that the mutation of the synthase, completely blocking motility (Figure 2) in LNLS-LB plates. On the contrary and in accordance with the higher production of AHLs that has been reported in low-salt LB liquid cultures in comparison with LNLS-LB (Mayer et al., 2018), in the case of the low-salt LB-plates, mixing the enzyme with the inoculum was not enough to block the motility, and it was necessary to mix the enzyme with all the culture media in order to fully abrogate this phenotype. At $37^{\circ} \mathrm{C}$ the abaI:Km mutant of $A$. nosocomialis M2 presented the same spreading phenotype as the parental strain in all culture media (Supplementary Figure 2).

\section{Biofilm Formation Is Under the Control of QS in A. baumannii ATCC ${ }^{\circledR}$ 17978 $^{\mathrm{TM}}$}

A preliminary experiment was performed in order to assess if low salinity promoted biofilm formation in A. baumannii ATCC ${ }^{\circledR}$ $17978^{\mathrm{TM}}$, as observed for surface-associated motility. Results 


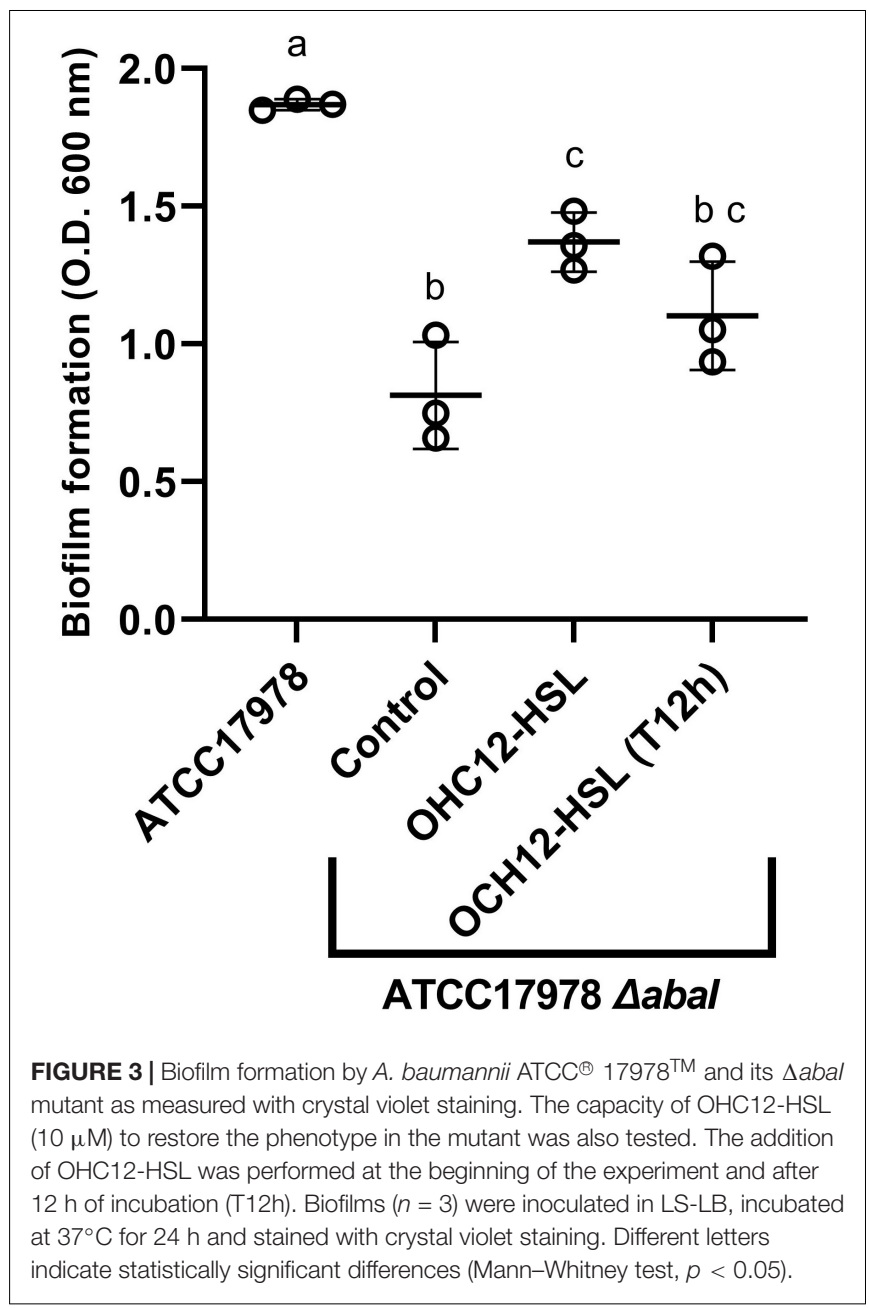

clearly show a significantly higher biofilm formation (MannWhitney test, $p<0.05$ ) in ATCC ${ }^{\circledast} 17978^{\mathrm{TM}}$ in the LS-LB medium in the first $12 \mathrm{~h}$, while no differences were observed thereafter with the crystal violet assay quantification (Supplementary Figure 4). The mutation of abaI gene caused a strong reduction in biofilm formation in A. baumannii ATCC ${ }^{\circledR} 17978^{\mathrm{TM}}$ in both culture media (Supplementary Figure 4). In an experiment performed in LS-LB, the addition of the QS signal OHC12-HSL to the mutant partially restored the phenotype, causing an increase of the biofilm biomass, that was statistically significant (MannWhitney test, $p<0.05$, Figure 3 ). The addition of the AHL to a preformed-biofilm (T12h) of the mutant also produced an increase in biofilm biomass, although this difference was not statistically significant (Mann-Whitney test, $p>0.05$ ).

\section{Effect of the QQ Enzyme Aii20J and Other Biofilm Inhibitory Enzymes on Biofilm Formation by $A$. baumannii ATCC $^{\circledR}$ 17978 $^{\text {TM }}$}

The purified QQ enzyme Aii20J reduced biofilm formation in A. baumannii $\mathrm{ATCC}^{\circledR} 17978^{\mathrm{TM}}$ by $80 \%$ after $24 \mathrm{~h}$ when grown on glass coverslips at $37^{\circ} \mathrm{C}$ (Figure 4). The enzyme also affected

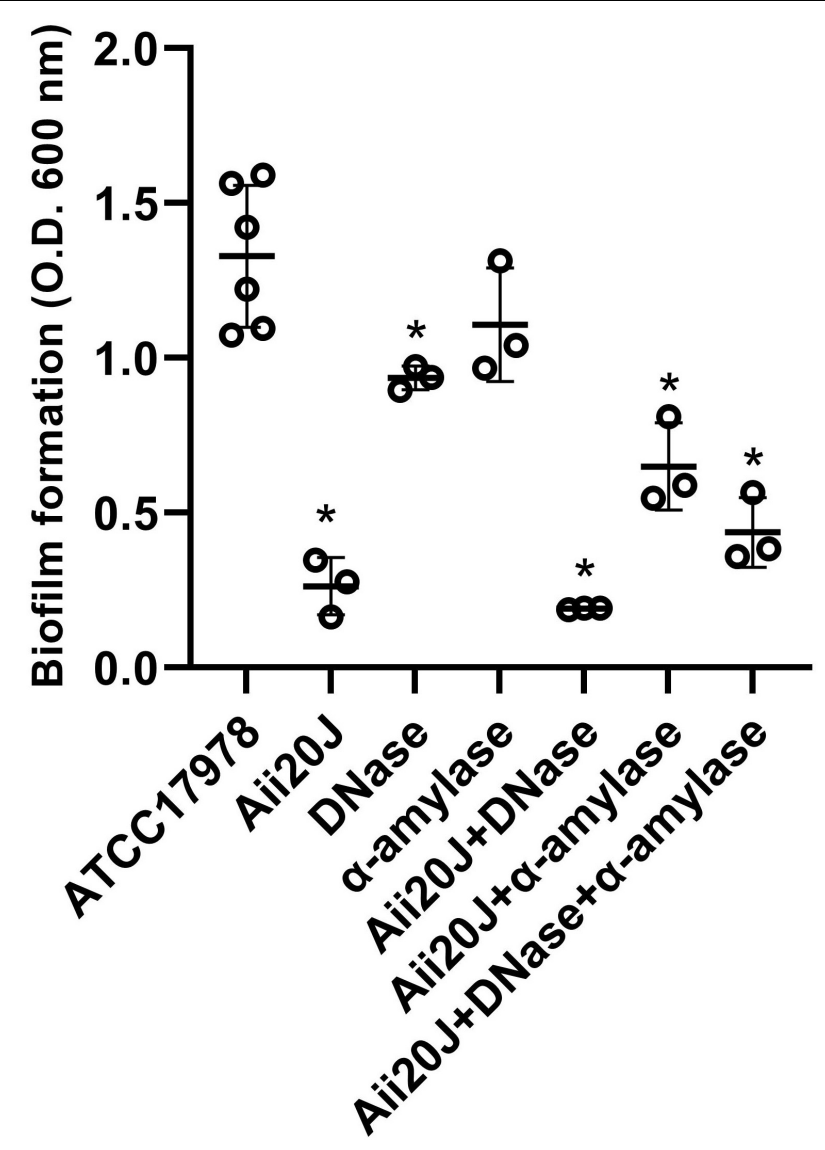

FIGURE 4 | Effect of different enzymes on biofilm formation by $A$. baumannii ATCC $^{\oplus} 17978^{\text {TM }}$. The QQ enzyme Aii20J $(20 \mu \mathrm{g} / \mathrm{mL})$, DNase $(2 \mathrm{U} / \mathrm{mL})$, and $\alpha$-amylase $(10 \mathrm{U} / \mathrm{mL})$ were added alone or in combination. Biofilms were produced in LS-LB in glass coverslips, incubated for $24 \mathrm{~h}$ at $37^{\circ} \mathrm{C}$ and stained with the crystal violet assay. The cultures were inoculated with cells from an agitated culture. Values are averages $\pm \operatorname{SD}(n=3)$. Asterisks indicate statistically significant differences in comparison with the untreated control (Mann-Whitney test, $p<0.05$ ).

biofilm formation in A. nosocomialis M2 in the same system, although the reduction was lower (38.54\%). The clinical strain MAR002 and an isogenic mutant impaired in biofilm formation and eukaryotic-cell attachment capacity were equally affected by the enzyme, suffering a reduction of biofilm formation of around 45\% (Supplementary Figure 5). Biofilm formation in MAR002 and its mutant was similar to that observed for ATCC ${ }^{\circledR}$ $17978^{\mathrm{TM}}$, even though MAR002 has been described as a biofilm hyper-forming strain (Álvarez-Fraga et al., 2016).

The effect of the purified QQ enzyme Aii20J was compared with the effect of two enzymes with potential biofilm inhibitory effect: a DNase and an $\alpha$-amylase. The $\alpha$-amylase did not affect biofilm formation by ATCC ${ }^{\circledR} 17978^{\mathrm{TM}}$ (Mann-Whitney test, $p=0.16$, Figure 4). On the contrary, DNase significantly reduced biofilm formation by $20 \%$ (Mann-Whitney test, $p<0.05$, Figure 4). In ATCC ${ }^{\circledR} 17978^{\mathrm{TM}}$ the simultaneous addition of the QQ enzyme Aii20J and DNase did not improve significantly 

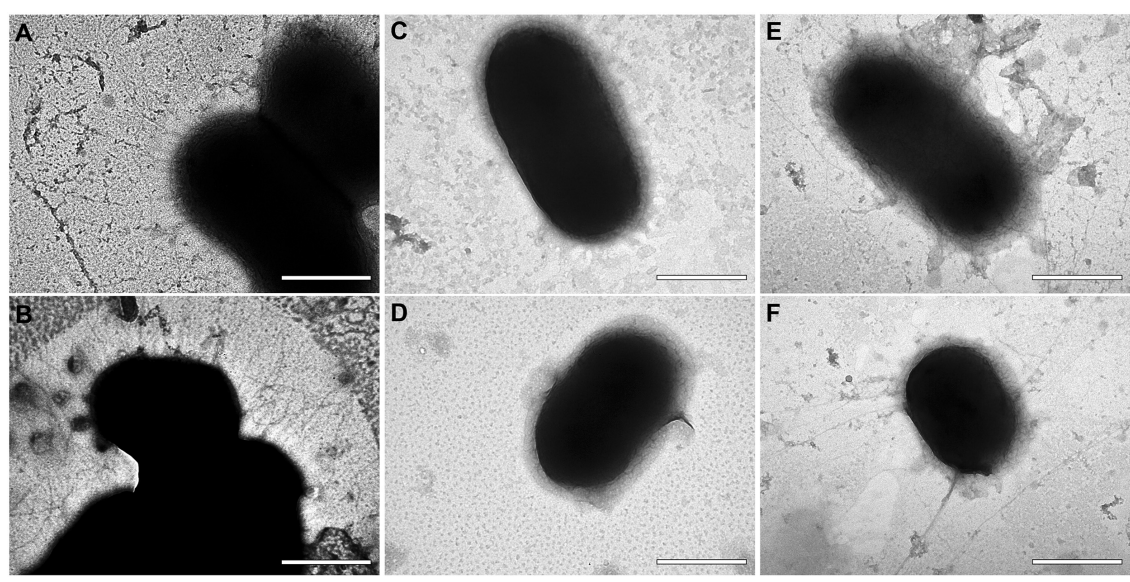

FIGURE 5 | TEM microphotographs of $A$. baumannii ATCC ${ }^{\circledR} 17978^{\text {TM }}$ (A,B), its isogenic mutant $\Delta$ abal $(\mathbf{C}, \mathbf{D})$ and $A$. baumannii ATCC $^{\circledR} 17978^{\text {TM }}$ treated with the QQ enzyme Aii20J (20 $\mu \mathrm{g} / \mathrm{mL})$ (E,F). Original magnification: $80.000 \times$. Scale bars: $500 \mathrm{~nm}$.

the results obtained with the QQ enzyme (Mann-Whitney test, $p=0.35$, Figure 4).

In order to observe the effect of Aii20J on cell appendages, biofilm and planktonic cells were observed with TEM. Important differences could be observed among wild type, the $\Delta a b a I$ mutant and the cells treated with the pure Aii20J enzyme (Figure 5). A. baumannii ATCC ${ }^{\circledR} 17978^{\mathrm{TM}}$ cells showed many short appendages (Figures 5A,B). On the contrary, cells of the $\triangle a b a I$ mutant and Aii20J-treated cells were almost completely devoid of these short appendages (Figures 5C-F). Cells surrounded by long, thin individual filaments were observed in the cultures in the presence of the Aii20J enzyme (Figures 5E,F). The same morphology was observed in attached and unattached cells (data not shown).

\section{Effect of the QQ Enzyme Aii20J and DNase on Biofilm Formation by Multidrug-Resistant Clinical Isolates of \\ A. baumannii}

Several clinical isolates that had been previously characterized regarding antibiotic-resistance profile, AHL production and surface-associated motility (López et al., 2017; Mayer et al., 2018) were selected and tested regarding its capacity to form biofilm on glass coverslips and their sensitivity to the enzymes Aii20J and DNase. Large differences in biofilm formation were found in all strains depending on the incubation conditions of the inoculum, with much higher biofilm formation being observed when the inoculum was maintained under static conditions (Figure 6B). Moreover, important differences among the tested strains were observed. A. baumannii ATCC $^{\circledR} 17978^{\mathrm{TM}}$ formed much more biofilm than any of the clinical MDR strains, independently of the culture conditions of the inoculum (Figures 6A,B). Among these, the biofilm formation capacity was not related to the antibiotic resistance or surface-associated motility profiles. Strains with high motility profile (Ab1, Ab5, Ab7) showed low or intermediate biofilm formation capacity in vitro. The effect of the enzymes was also variable among the strains (Figures $6 \mathbf{A}, \mathbf{B}$ ). However, no significant effect on bacterial growth, measured as optical density in the supernatant of the cultures was observed, except for ATCC17978 in which OD in the supernatants in the presence of the enzymes was significantly higher than in the control culture (data not shown). Strains ATCC $^{\circledR} 17978^{\mathrm{TM}}$ and Ab7, showing a very similar surface-associated motility pattern and response to the QQ enzyme (López et al., 2017) showed a very different response regarding biofilm formation. Best inhibitory results were obtained with the combination of the QQ enzyme and DNase in all cases (Figures 6A,B).

\section{DISCUSSION}

The study of the factors controlling the expression of virulence factors is crucial for the development of novel antimicrobial strategies in antibiotic-resistant pathogens like A. baumannii. Multiple transcriptional regulatory systems are involved in the persistence and pathogenesis of this species (Wood et al., 2018a; De Silva and Kumar, 2019). Among these regulatory circuits, QS has emerged as an interesting target for the control of virulence factors (Zhang et al., 2017; Mayer et al., 2018). Most studies regarding the role of the AHL-mediated QS system in the expression of virulence-related traits have been carried out with A. nosocomialis strain M2 (formerly A. baumannii M2) (Niu et al., 2008; Bhargava et al., 2010; Clemmer et al., 2011; Stacy et al., 2012, 2013; Saroj and Rather, 2013; Oh and Choi, 2015). The role of the QS system in this intricate signaling system seems to be crucial, since the mutation of the AbaI synthase significantly reduces virulence in in vivo models (Peleg et al., 2009; Fernández-García et al., 2018). Nevertheless, in the view that some important features, such as motility, behave differently between A. nosocomialis M2 and A. baumannii ATCC $^{\circledR} 17978^{\mathrm{TM}}$ (Clemmer et al., 2011), it is important to fully characterize the role of QS system in the latter and to study the variations among clinical strains of A. baumannii. As previously reported for other 


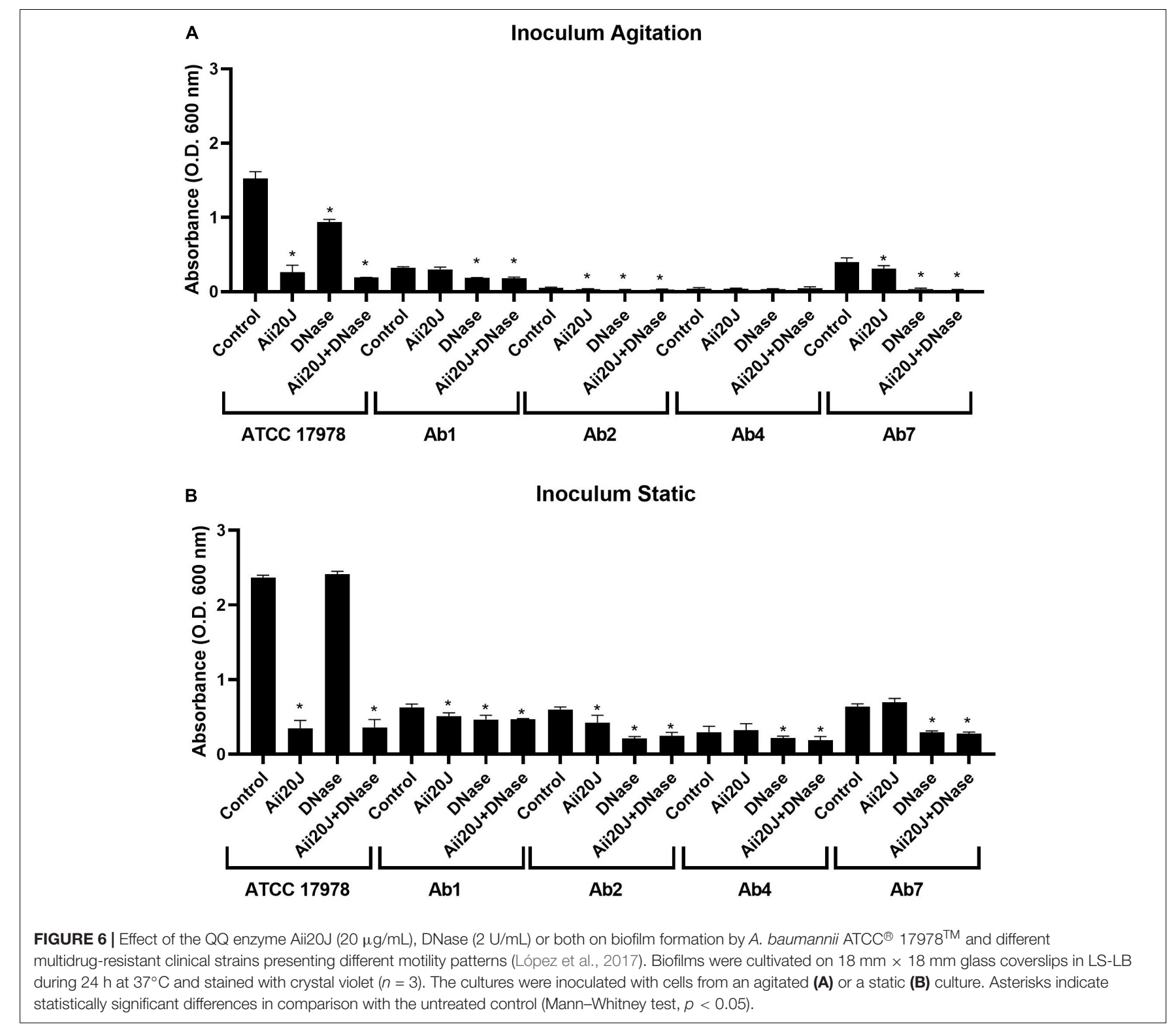

species of the genus (Niu et al., 2008; Bitrian et al., 2012; How et al., 2015), the mutation of the abaI gene (A1S_0109) fully abolished AHL production in A. baumannii ATCC ${ }^{\circledR} 17978^{\mathrm{TM}}$, indicating that this gene probably codifies the only synthase responsible for AHL-mediated QS in this species.

Both, motility (Figures 1, 2) and biofilm formation (Figure 3) were impaired in the $\triangle a b a I$ mutant in $A$. baumannii ATCC $^{\circledR}$ $17978^{\mathrm{TM}}$, corroborating previous reports on the control of these two important traits related to virulence by the AHLmediated QS system in other Acinetobacter spp. (Niu et al., 2008; Kang and Park, 2010; Anbazhagan et al., 2012; Bhargava et al., 2012; Kim and Park, 2013; Chow et al., 2014; Oh and Choi, 2015; Mayer et al., 2018). The exogenous addition of OHC12HSL, the main AHL found in Acinetobacter spp., restored the motility of the $\triangle a b a I$ mutant under motile-permissive, low-salt conditions (Figure 1) and partially restored biofilm forming capacity (Figure 3). Consistent with these outcomes, in ATCC $^{\circledR} 17978^{\mathrm{TM}}$ the wide-spectrum AHL-lactonase Aii20J blocked motility even in highly inductive conditions (Figure 2). Moreover, it drastically reduced biofilm formation (Figures 4, 6 and Supplementary Figure 5), confirming a key role of QS in the regulation of these two traits in this strain and further supporting the potential of QQ strategies for virulence control in Acinetobacter (Stacy et al., 2013; Chow et al., 2014; Mayer et al., 2015, 2018, 2020; López et al., 2017; Zhang et al., 2017). Nevertheless, a wide variability in surface-associated motility and response to QQ strategies has been previously described in A. baumannii clinical isolates presenting different antibiotic resistance profiles (López et al., 2017). Also, the well-studied strain A. nosocomialis M2 presented very different behavior in response to culture conditions and QQ strategies in comparison with ATCC $^{\circledR} 17978^{\mathrm{TM}}$, maintaining a strong motility phenotype 
in conditions in which $A$. baumannii ATCC ${ }^{\circledR} 17978^{\mathrm{TM}}$ motility was completely blocked (Supplementary Figure 2). Unlike what was observed for the QS-impaired mutant of ATCC ${ }^{\circledR} 17978^{\mathrm{TM}}$, the A. nosocomialis M2 abaI:Km mutant maintained the motility pattern at $37^{\circ} \mathrm{C}$ in contrast with the impairment of motility reported at $30^{\circ} \mathrm{C}$ (Clemmer et al., 2011) and confirmed in our laboratory (data not shown), indicating a strong effect of temperature in this type of in vitro assays. Our results demonstrate that surface-associated motility in M2 is not so strongly dependent on QS regulatory pathways, at least in motilepermissive conditions, revealing important differences in the role of QS on this phenotype among species and isolates of the genus Acinetobacter.

High salinity negatively affected both surface-associated motility and initial biofilm formation in ATCC $^{\circledR} 17978^{\mathrm{TM}}$ (Figure 2 and Supplementary Figure 4). Salinity lost its influence during biofilm maturation under the conditions tested (Figure 2), indicating that the factors that favor motility at low salinities are important for the initial steps of biofilm formation, and linking the mechanisms responsible for surface-associated motility with the surface-attachment phase of biofilm formation. Indeed, AHL production is higher in low-salinity liquid cultures (Mayer et al., 2018), which also correlates QS with these two physiological processes. In low-salt solid cultures it was necessary to increase the concentration of the QQ enzyme Aii20J in order to reduce motility, indicating a higher production of QS signals also in semi-solid LS-LB. High osmolarity was shown to repress motility but to activate biofilm formation in Vibrio cholerae (Shikuma and Yildiz, 2009), suggesting that medium osmolarity is an important environmental signal regulating lifestyle in biofilmforming pathogens.

The electron microscope analysis of cells obtained during early biofilm formation shows a clear decrease in surface short pili in the $\triangle a b a I$ mutant (Figure 5), which could be the result of lower expression of the csu operon. The chaperone/usher pili-associated csu operon, responsible for the assembly and extension of type I pilus (Tomaras et al., 2003, 2008), important for biofilm formation in A. nosocomialis M2 and A. baumannii ATCC $^{\circledR} 19606^{\text {TM }}$ (Tomaras et al., 2008; Gaddy and Actis, 2009; Clemmer et al., 2011; Luo et al., 2015), has been previously described to be overexpressed in biofilms in comparison with planktonic cultures of $A$. baumannii ATCC $^{\circledR} 17978^{\mathrm{TM}}$ (RumboFeal et al., 2013). The $c s u$ operon has also been related to surfaceassociated motility in A. baumannii ATCC ${ }^{\circledR} 17978^{\mathrm{TM}}$ since the conditions that promote motility and AHL signal production also generate an increase in the expression of $\operatorname{csuD}$ (Mayer et al., 2018). The mutation of A1S_2811, a CheA/Y-like hybrid, a twocomponent regulator in A. baumannii $\mathrm{ATCC}^{\circledR} 17978^{\mathrm{TM}}$, resulted in a decrease in surface motility and biofilm formation at the gas-liquid interface and the transcription of $a b a I$ and the csu operon. The reduction of biofilm and motility in the mutant was accompanied by a decrease in pilus-like structures and exopolymeric substances on the cell surface (Chen et al., 2017). Moreover, Luo et al. (2015) showed that addition of AHL signals to A. baumannii ATCC19606 ${ }^{\mathrm{TM}}$ cultures induces the expression of bacterial pili structural genes, including $\operatorname{csuD}$, and an increase of pili-like structures around the bacteria. Our results further support a role of the csu operon in surface-associated motility and biofilm formation, both being under the control of the QS signals in ATCC $^{\circledR} 17978^{\mathrm{TM}}$. Consistent with a control of $c s u$ expression by the QS system, short pili were also absent in cells treated with the QQ enzyme Aii20J; instead, long filaments were found around the cells treated with the enzyme, that were absent in the wild type and in the $\triangle a b a I$ mutant (Figure 5), indicating that the QQ enzyme could not block the production of a different type appendages. These filaments could be attributed, among others, to type IV pili (TFP), involved in several bacterial processes such as natural transformation or adherence to biotic and abiotic surfaces and twitching motility (Clemmer et al., 2011; Harding et al., 2013; Eijkelkamp et al., 2014). However, the involvement of TFP in surface-associated motility and its regulation by the QS system is controversial (reviewed by Harding et al., 2018). A novel photo-regulated type I chaperone/usher pilus assembly system, encoded by the prpABCD operon, involved in surface-associated motility, biofilm formation and virulence to Galleria mellonella (Wood et al., 2018b) could be also responsible for the observed phenotype in the Aii20J-treated cells. Additionally, the synthesis of 1,3-diaminopropane (DAP), lipid oligosaccharides, other external envelope components or extracellular DNA, important for motility and biofilm formation in this species (McQueary et al., 2012; Harding et al., 2018; Geisinger et al., 2019) may also be under the control of the QS system, contributing to the observed effect of the mutation of the $a b a \mathrm{I}$ gene and Aii20J lactonase on both, surface-associated motility and biofilm formation.

The status of the inoculum strongly influenced the formation of biofilm in the Amsterdam Active Attachment model (Figure 6). More biofilm was formed when the inoculum was maintained in static conditions, a phenomenon already reported for pellicle formation in the liquid-air interface and motility in A. baumannii ATCC ${ }^{\circledR} 17978^{\text {TM }}$ (Chen et al., 2017). This fact also links biofilm formation with the QS system, since AHLs are only produced in significant amounts in static cultures (Mayer et al., 2018). Therefore, the cells maintained in such conditions should display a set of physiological activities that favor or accelerate the initiation of biofilm formation when transferred to fresh medium.

As previously reported for surface-associated motility (López et al., 2017), strong variations in biofilm formation were found among the different strains tested, and no clear correlation was found between strain origin, antibiotic resistance profile and in vitro biofilm formation ability (Figure 6). A large number of works have analyzed the correlation between biofilm formation and antibiotic resistance in A. baumannii (reviewed by Eze et al., 2018; Mayer et al., 2020). Biofilm formation has been found to be correlated with MDR among clinical isolates (Babapour et al., 2016; Yang et al., 2019; Zeighami et al., 2019). Higher incidence of antibiotic resistance has also been associated with the presence of $a b a I$ and $c s u E$ genes in clinical A. baumannii strains (Liu et al., 2016). On the contrary, another study could not find any relationship between isolation site, multidrug resistance, pulsed-field type and biofilm production among A. baumannii clinical and environmental strains isolated from a hospital (de Campos et al., 2016). Finally, it was reported that isolates with a higher level of resistance tended to form weaker biofilms (Qi et al., 2016) driving to the hypothesis that biofilm-forming 
bacterial strains do not depend on antibiotic resistance and colonization characteristics as do non-biofilm formers for their survival in hospital settings (Hall and Mah, 2017). In our case, the low-antibiotic resistant strain $\mathrm{ATCC}^{\circledR} 17978^{\mathrm{TM}}$, isolated from a case of infant meningitis, shows a much higher biofilm-forming capacity than the MDR strains (Figure 6).

The QQ lactonase Aii20J strongly reduced biofilm formation in A. baumannii ATCC ${ }^{\circledR} 17978^{\mathrm{TM}}$. This fact indicates that the many existing QQ enzymes present in this strain may be active only under particular conditions or during specific periods, probably related to the self-control of AHL production during stationary phase (Mayer et al., 2018). The lactonase was also capable of reducing biofilm formation in A. nosocomialis M2 and in MAR002, that was previously reported as a biofilm hyper-forming strain, even though in our cultivation system the amount of biofilm formed was similar to that observed in A. baumannii ATCC ${ }^{\circledR} 17978^{\mathrm{TM}}$ (Supplementary Figure 5). This difference is probably the result of the different cultivation methodology and culture medium used, since in tubes, these strains form biofilm only in the interphase liquid:air (Tomaras et al., 2003). The lactonase Aii20J also reduced biofilm formation significantly in two of the four MDR clinical strains tested (Figure 6). Sensitivity to the enzymes in the clinical MDR strains was not dependent of MDR profile or biofilm-forming capacity. However, it could be somehow related to the amount of AHLs produced since strains $\mathrm{Ab} 5$ and $\mathrm{Ab} 7$ produced a negligible amount of AHLs in LB medium at $37^{\circ} \mathrm{C}$ (Mayer et al., 2018) and demonstrated no-sensitivity to the action of the QQ enzyme (Figure 6). A recombinant lactonase successfully disrupted the biofilm formation of clinical isolates of $A$. baumannii (Chow et al., 2014). However, MomL was ineffective in treating mixed species and wound-associated biofilm models containing A. baumannii (Zhang et al., 2017). The differences observed in biofilm formation when the inoculum was maintained in static or shaken conditions indicate that the published results regarding biofilm formation may be strongly influenced by cultivation conditions and should be interpreted carefully. Temperatures lower than $30^{\circ} \mathrm{C}$ seem to favor biofilm formation (Eze and El Zowalaty, 2019), and QS regulated genes that are relevant for biofilm formation are upregulated at lower temperatures (De Silva and Kumar, 2019). Current biofilm experiments were carried out at $37^{\circ} \mathrm{C}$, and therefore the effect of the QQ lactonase Aii20J may be higher at lower temperatures. Also, the weak biofilm formation by this species in the microtiterplate method generally used to cultivate the biofilms, and the low sensitivity of the crystal-violet-staining method used for the assessment of biofilm biomass in most studies should be taken into account when evaluating anti-biofilm activity (Muras et al., 2018).

Despite the variability in response to the QQ lactonase Aii20J and DNase, the simultaneous addition of both enzymes reduced biofilm formation in the five strains tested, and therefore constitutes a promising strategy for controlling biofilm formation in this species. The presence of eDNA in A. baumannii biofilms has been reported before in a clinical isolate and a reduction of biofilm formation by DNase I has been already reported, even in preformed biofilms (Tetz et al., 2009; Sahu et al.,
2012). Since the sequence of eDNA is similar to genomic DNA, the production of eDNA may also constitute a way of transferring antibiotic resistance in this species (Li et al., 2008). eDNA has been reported in numerous Gram-positive and Gram-negative bacterial pathogens, and the efficacy of the use of DNase has been demonstrated in many of them, increasing the efficacy of antibiotics (Tetz et al., 2009; Okshevsky et al., 2015). In our case, the efficacy of DNase to reduce biofilm formation in A. baumannii ATCC ${ }^{\circledR} 17978^{\mathrm{TM}}$ and different clinical isolates was highly strain-dependent (Figure 6). In the view of the obtained results, the pathway of eDNA secretion in A. baumannii deserves further investigation since it constitutes a promising target for developing combined antibiofilm strategies.

Based on the study of the $\triangle a b a I$ mutant and the action of the QQ enzyme Aii20J, results confirm a central role of the QS in the control of biofilm formation and surfaceassociated motility in A. baumannii ATCC ${ }^{\circledR} 17978^{\mathrm{TM}}$. This role seems to be strain-specific and strongly dependent on the cultivation conditions. Results demonstrate that QQ strategies, in combination with other enzymatic treatments such as DNase, could represent an alternative approach for the prevention of A. baumannii colonization and survival of surfaces and the prevention and treatment of infections caused by this pathogen. The wide variability observed in surface-associated motility, biofilm forming capacity and sensitivity to the QQ enzyme among the tested $A$. baumannii isolates indicate that the development of any novel antimicrobial strategy targeting virulence traits should be carefully evaluated on a large number of strains.

\section{DATA AVAILABILITY STATEMENT}

All datasets generated for this study are included in the article/Supplementary Material.

\section{AUTHOR CONTRIBUTIONS}

CM, AM, and AP conducted the experiments and contributed to the writing of the manuscript. SR-F constructed the mutant. JR-V performed the TEM analysis and contributed to the writing of the manuscript. MR and MP contributed to the writing of the manuscript. AO designed the experiments and wrote the manuscript. All authors contributed to the article and approved the submitted version.

\section{FUNDING}

AM was supported by a predoctoral fellowship from Xunta de Galicia (ED481A-2015/311). CM was supported by a postdoctoral fellowship from Xunta de Galicia (IN606B-2019/010). MR was supported by the Biotechnology and Biological Sciences Research Council (BB/R012415/1). AP was supported by a predoctoral fellowship from Xunta de Galicia (ED481A-2019/194). 


\section{ACKNOWLEDGMENTS}

We thank Prof. Philip N. Rather from the Emory University School of Medicine of Atlanta (United States) for kindly providing us with the abaI:Km mutant strain of A. nosocomialis M2. The clinical strains of A. baumannii were provided by Dr. María Tomás Carmona (CHUACINIBIC, A Coruña, Spain) on behalf of the GEIHGEMARA (SEIMC).

\section{SUPPLEMENTARY MATERIAL}

The Supplementary Material for this article can be found online at: https://www.frontiersin.org/articles/10.3389/fmicb. 2020.565548/full\#supplementary-material

Supplementary Figure 1 | Effect of culture media composition and type of agar on surface-associated motility of $A$. baumannii ATCC17978, A. nosocomialis M2 and their respective mutants of the Abal AHL-synthase. LB and Low Salt-Low Nutrient LB (LSLN-LB $0.5 \% \mathrm{NaCl}, 0.2 \%$ tryptone, and $0.1 \%$ yeast extract) were tested. Eiken or Difco agar were added at $0.25 \%$. Plates were incubated at $37^{\circ} \mathrm{C}$ for $14 \mathrm{~h}$, and they are representative of three independent experiments carried out with three replicates per condition.

Supplementary Figure 2 | Effect of the components of LB culture medium on the motility of $A$. nosocomialis M2 and its abal: Km mutant. $0.25 \%$ Eiken agar

\section{REFERENCES}

Álvarez-Fraga, L., Pérez, A., Rumbo-Feal, S., Merino, M., Vallejo, J. Á, Ohneck, E. J., et al. (2016). Analysis of the role of the LH92_11085 gene of a biofilm hyper-producing Acinetobacter baumannii strain on biofilm formation and attachment to eukaryotic cells. Virulence 7, 443-445. doi: 10.1080/21505594. 2016.1145335

Anbazhagan, D., Mansor, M., Yan, G. O., Md Yusof, M. Y., Hassan, H., and Sekaran, S. D. (2012). Detection of quorum sensing signal molecules and identification of an autoinducer synthase gene among biofilm forming clinical isolates of Acinetobacter spp. PLoS One 7:e36696. doi: 10.1371/journal.pone.0036696

Arivett, B. A., Fiester, S. E., Ream, D. C., Centrón, D., Ramírez, M. S., Tolmasky, M. E., et al. (2015). Draft Genome of the multidrug-resistant Acinetobacter baumannii strain A155 clinical isolate. Genome Announc. 3, e212-e215. doi: 10.1128/genomeA.00212-15

Babapour, E., Haddadi, A., Mirnejad, R., Angaji, S.-A., and Amirmozafari, N. (2016). Biofilm formation in clinical isolates of nosocomial Acinetobacter baumannii and its relationship with multidrug resistance. Asian Pac. J. Trop. Biomed. 6, 528-533. doi: 10.1016/j.apjtb.2016.04.006

Bhargava, N., Sharma, P., and Capalash, N. (2010). Quorum sensing in Acinetobacter: an emerging pathogen. Crit. Rev. Microbiol. 36, 349-360. doi: 10.3109/1040841X.2010.512269

Bhargava, N., Sharma, P., and Capalash, N. (2012). N-acyl homoserine lactone mediated interspecies interactions between A. baumannii and P. aeruginosa. Biofouling 28, 813-822. doi: 10.1080/08927014.2012.714372

Bitrian, M., Solari, C. M., González, R. H., and Nudel, C. B. (2012). Identification of virulence markers in clinically relevant strains of Acinetobacter genospecies. Int. Microbiol. 15, 79-88.

Carruthers, M. D., Harding, C. M., Baker, B. D., Bonomo, R. A., Hujer, K. M., Rather, P. N., et al. (2013). Draft genome sequence of the clinical isolate Acinetobacter nosocomialis strain M2. Genome Announc. 1, e906-e913. doi: 10.1128/genomeA.00906-13

Chan, K. G., Atkinson, S., Mathee, K., Sam, C. K., Chhabra, S. R., Cámara, M., et al. (2011). Characterization of $\mathrm{N}$-acylhomoserine lactone-degrading bacteria associated with the Zingiber officinale (ginger) rhizosphere: co-existence of plates were incubated at $37^{\circ} \mathrm{C}$ for $14 \mathrm{~h}$. Images are representative of three independent experiments carried out with three replicates per condition.

Supplementary Figure 3 | Effect of osmolarity on surface-associated motility in A. baumannii ATCC ${ }^{\circledR} 17978^{\mathrm{TM}} \mathbf{( A )}$ and A. nosocomialis M2 (B). Cells were inoculated on LB plates with different $\mathrm{NaCl}(0.1-0.4 \mathrm{M})$ or sucrose (5-20\%) concentrations. Plates were incubated at $37^{\circ} \mathrm{C}$ for $14 \mathrm{~h}$. Images are representative of three independent experiments carried out carried out with three replicates per condition. $\mathrm{NaCl}$ concentration in standard LB medium is $0.17 \mathrm{M}$.

Supplementary Figure 4 | Biofilm formation by A. baumannii ATCC ${ }^{\oplus} 17978^{\text {TM }}$ and its isogenic mutant $\triangle a b a l$ in LB (gray bars) and low-salt LB (LS-LB, NaCl $0.5 \%$, white bars) culture media. Biofilms were formed on $18 \mathrm{~mm} \times 18 \mathrm{~mm}$ glass coverslips using a modification of the Active Attachment model (Exterkate et al., 2010), incubated for $24 \mathrm{~h}$ at $37^{\circ} \mathrm{C}$ and quantified using the crystal violet assay. Values are shown as average $\pm \operatorname{SD}(n=3)$. The asterisk shows statistically significant differences (Mann-Whitney test, $p<0.05$ ) between LB and LS-LB.

Supplementary Figure 5 | Effect of the QQ lactonase Aii20J $(20 \mu \mathrm{g} / \mathrm{mL})$ on biofilm formation by $A$. baumannii ATCC ${ }^{\circledR} 17978^{\mathrm{TM}}$, the $A$. baumannii clinical strain MAR002, which was reported to be a biofilm hyper-former, its isogenic mutant $\Delta 11085$, which was reported to be defective in biofilm formation and eukaryotic cell attachment in comparison with the parental strain (Álvarez-Fraga et al., 2016) and A. nosocomialis M2. (A) Biofilm quantification using the Crystal Violet staining method. Values are averages $\pm \mathrm{SD}(n=3)$. The asterisks show statistically significant differences (Mann-Whitney test, $p<0.05$ ) between treated and untreated biofilms for the same strain. (B) Pictures showing the aspect of the untreated (left) and treated (right) biofilms stained with crystal violet. Biofilms were produced on $18 \mathrm{~mm} \times 18 \mathrm{~mm}$ glass coverslips using a modification of the Amsterdam Active Attachment model (Exterkate et al., 2010) in LS-LB, incubated for $24 \mathrm{~h}$ at $37^{\circ} \mathrm{C}$.

quorum quenching and quorum sensing in Acinetobacter and Burkholderia. BMC Microbiol. 11:51. doi: 10.1186/1471-2180-11-51

Chan, K. G., Cheng, H. J., Chen, J. W., Yin, W. F., and Ngeow, Y. F. (2014). Tandem mass spectrometry detection of quorum sensing activity in multidrug resistant clinical isolate Acinetobacter baumannii. Scientific WorldJournal 2014:891041. doi: 10.1155/2014/891041

Chen, R., Lv, R., Xiao, L., Wang, M., Du, Z., Tan, Y., et al. (2017). A1S_2811, a CheA/Y-like hybrid two-component regulator from Acinetobacter baumannii ATCC17978, is involved in surface motility and biofilm formation in this bacterium. Microbiologyopen 6:e00510. doi: 10.1002/mbo3.510

Chow, J. Y., Yang, Y., Tay, S. B., Chua, K. L., and Yew, W. S. (2014). Disruption of biofilm formation by the human pathogen Acinetobacter baumannii using engineered quorum-quenching lactonase. Antimicrob. Agent Chemother. 58, 1802-1805. doi: 10.1128/AAC.02410-13

Clemmer, K. M., Bonomo, R. A., and Rather, P. N. (2011). Genetic analysis of surface motility in Acinetobacter baumannii. Microbiology 157, 2534-2544. doi: 10.1099/mic.0.049791-0

de Campos, P. A., Royer, S., da Fonseca Batistão, D. W., Araújo, B. F., Queiroz, L. L., de Brito, C. S., et al. (2016). Multidrug resistance related to biofilm formation in Acinetobacter baumannii and Klebsiella pneumonia clinical strains from different pulsotype. Curr. Microbiol. 72, 617-627. doi: 10.1007/s00284016-0996-x

De Silva, P. M., and Kumar, A. (2019). Signal transduction proteins in Acinetobacter baumannii: role in antibiotic resistance, virulence, and potential as drug targets. Front. Microbiol. 10:49. doi: 10.3389/fmicb.2019.00049

Eijkelkamp, B. A., Stroeher, U. H., Hassan, K. A., Elbourne, L. D., Paulsen, I. T., and Brown, M. H. (2013). H-NS plays a role in expression of Acinetobacter baumannii virulence features. Infect. Immun. 81, 2574-2783. doi: 10.1128/IAI. 00065-13

Eijkelkamp, B. A., Stroeher, U. H., Hassan, K. A., Papadimitrious, M. S., Paulsen, I. T., and Brown, M. H. (2011). Adherence and motility characteristics of clinical Acinetobacter baumannii isolates. FEMS Microbiol. Lett. 323, 44-51. doi: 10.1111/j.1574-6968.2011.02362.x

Eijkelkamp, B. A., Stroeher, U. H., Hassan, K. A., Paulsen, I. T., and Brown, M. H. (2014). Comparative analysis of surface-exposed virulence factors of 
Acinetobacter baumannii. BMC Genomics 15:1020. doi: 10.1186/1471-2164-151020

Exterkate, R. A., Crielaard, W., and Ten Cate, J. M. (2010). Different response to amine fluoride by Streptococcus mutans and polymicrobial biofilms in a novel high-throughput active attachment model. Caries Res. 44, 372-379. doi: $10.1159 / 000316541$

Eze, E. C., Chenia, H. Y., and El Zowalaty, M. E. (2018). Acinetobacter biofilms: effects of physicochemical factors, virulence, antibiotic resistance determinants, gene regulation, and future antimicrobial treatments. Infect. Drug Resist. 11, 2277-2299. doi: 10.2147/IDR.S169894

Eze, E. C., and El Zowalaty, M. E. (2019). Combined effect of low incubation temperature, minimal growth medium, and low hydrodynamics optimize Acinetobacter baumannii biofilm formation. Infect. Drug Resist. 12, 3523-3536. doi: 10.2147/IDR.S203919

Fernández-García, L., Ambroa, A., Blasco, L., Bleriot, I., López, M., AlvarezMarin, R., et al. (2018). Relationship between the quorum network (sensing/quenching) and clinical features of pneumonia and bacteraemia caused by A. baumannii. Front. Microbiol. 9:3105. doi: 10.3389/fmicb.2018. 03105

Gaddy, J. A., and Actis, L. A. (2009). Regulation of Acinetobacter baumannii biofilm formation. Future Microbiol. 4, 273-278. doi: 10.2217/fmb.09.5

Geisinger, E., Huo, W., Hernandez-Bird, J., and Isberg, R. R. (2019). Acinetobacter baumannii: envelope determinants that control drug resistance, virulence, and surface variability. Annu. Rev. Microbiol. 73, 481-506. doi: 10.1146/annurevmicro-020518-115714

Giles, S. K., Stroeher, U. H., Eijkelkamp, B. A., and Brown, M. H. (2015). Identification of genes essential for pellicle formation in Acinetobacter baumannii. BMC Microbiol. 15:116. doi: 10.1186/s12866-015-0440-6

Hall, C. W., and Mah, T. F. (2017). Molecular mechanisms of biofilm-based antibiotic resistance and tolerance in pathogenic bacteria. FEMS Microbiol. Lett. Rev. 41, 276-301. doi: 10.1093/femsre/fux010

Hamad, M. A., Zajdowicz, S. L., Holmes, R. K., and Voskuil, M. I. (2009). An allelic exchange system for compliant genetic manipulation of the select agents Burkholderia pseudomallei and Burkholderia mallei. Gene 430, 123-131.

Harding, C. M., Hennon, S. W., and Feldman, M. F. (2018). Uncovering the mechanisms of Acinetobacter baumannii virulence. Nat. Rev. Microbiol. 16, 91-102. doi: 10.1038/nrmicro.2017.148

Harding, C. M., Tracy, E. N., Carruthers, M. D., Rather, P. N., Actis, L. A., and Munson, R. S. Jr. (2013). Acinetobacter baumannii strain M2 produces type IV pili which play a role in natural transformation and twitching motility but not surface-associated motility. mBio 4:e00360-13. doi: $10.1128 / \mathrm{mBio} .00$ 360-13

How, K. Y., Hong, K. W., Sam, C. K., Koh, C. L., Yin, W. F., and Chan, K. G. (2015). Unravelling the genome of long chain $\mathrm{N}$-acylhomoserine lactone-producing Acinetobacter sp. strain GG2 and identification of its quorum sensing synthase gene. Front. Microbiol. 6:240. doi: 10.3389/fmicb.2015.00240

Kang, B. R., Lee, J. H., Ko, S. J., Lee, Y. H., Cha, J. S., Cho, B. H., et al. (2004). Degradation of acyl-homoserine lactone molecules by Acinetobacter sp. strain C1010. Can. J. Microbiol. 50, 935-941.

Kang, Y. S., and Park, W. (2010). Contribution of quorum-sensing system to hexadecane degradation and biofilm formation in Acinetobacter sp. strain DR1. J. Appl. Microbiol. 109, 1650-1659. doi: 10.1111/j.1365-2672.2010.04 793.x

Kim, A. L., Park, S. Y., Lee, C. H., Lee, C. H., and Lee, J. K. (2014). Quorum quenching bacteria isolated from sludge of a wastewater treatment plant and their application for controlling biofilm formation. J. Microbiol. Biotechnol. 24, 1574-1582.

Kim, J., and Park, W. (2013). Identification and characterization of genes regulated by AqsR, a LuxR-type regulator in Acinetobacter oleivorans DR1. Appl. Microbiol. Biotechnol. 97, 6967-6978. doi: 10.1007/s00253-013-50065007

Lee, C. R., Lee, J. H., Park, M., Park, K. S., Bae, I. K., Kim, Y. B., et al. (2017). Biology of Acinetobacter baumannii: pathogenesis, antibiotic resistance mechanisms, and prospective treatment options. Front. Cell. Infect. Microbiol. 7:55. doi: 10. 3389/fcimb.2017.00055

Li, L., Xia, Z., Hu, Z., Zhou, Z., and Li, H. (2008). Expression of class I integrase gene in Acinetobacter baumannii and drug-resistance. Zhong Nan Da Xue Xue Bao Yi Xue Ban 33, 952-957.
Liu, H., Wu, Y. Q., Chen, L. P., Gao, X., Huang, H. N., Qiu, F. L., et al. (2016). Biofilm-related genes: analyses in multi-antibiotic resistant Acinetobacter baumannii isolates from mainland China. Med. Sci. Monit. 22:1801.

López, M., Mayer, C., Fernández-García, L., Blasco, L., Muras, A., Ruiz, F. M., et al. (2017). Quorum sensing network in clinical strains of A. baumannii: AidA is a new quorum quenching enzyme. PLoS One 12:e0174454. doi: 10.1371/journal. pone. 0174454

Luo, L. M., Wu, L. J., Xiao, Y. L., Zhao, D., Chen, Z. X., Kang, M., et al. (2015). Enhancing pili assembly and biofilm formation in Acinetobacter baumannii ATCC19606 using non-native acyl-homoserine lactones. BMC Microbiol. 15:62. doi: 10.1186/s12866-015-0397-395

Marti, S., Nait Chabane, Y., Alexandre, S., Coquet, L., Vila, J., Jouenne, T., et al. (2011). Growth of Acinetobacter baumannii in pellicle enhanced the expression of potential virulence. PLoS One 6:e26030. doi: 10.1371/journal.pone.0026030

Mayer, C., Muras, A., Romero, M., López, M., Tomás, M., and Otero, A. (2018). Multiple quorum quenching enzymes are active in the nosocomial pathogen Acinetobacter baumannii ATCC17978. Front. Cell. Infect. Microbiol. 8:310. doi: $10.3389 /$ fcimb.2018.00310

Mayer, C., Romero, M., López, M., Muras, A., and Otero, A. (2020). Quorum Sensing in Acinetobacter Virulence. Washington, DC: ACS Books.

Mayer, C., Romero, M., Muras, A., and Otero, A. (2015). Aii20J, a widespectrum termostable $N$-acylhomoserine lactonase from the marine bacterium Tenacibaculum sp. 20J, can quench AHL-mediated acid resistance in Escherichia coli. Appl. Microbiol. Biotechnol. 99, 9523-9539. doi: 10.1007/s00253-0156741-8

McQueary, C. N., Kirkup, B. C., Si, Y., Barlow, M., Actis, L. A., Craft, D. W., et al. (2012). Extracellular stress and lipopolysaccharide modulate Acinetobacter surface-associated motility. J. Microbiol. 50, 434-443. doi: 10.1007/s12275-0121555-1

Moubareck, C. A., and Halat, D. H. (2020). Insights into Acinetobacter baumannii: a review of microbiological, virulence, and resistance traits in a threatening nosocomial pathogen. Antibiotics 9:119. doi: 10.3390/antibiotics9030119

Muras, A., Mayer, C., Romero, M., Camino, T., Ferrer, M. D., Mira, A., et al. (2018). Inhibition of Streptococcus mutans biofilm formation by extracts of Tenacibaculum sp. 20J, a bacterium with wide-spectrum quorum quenching activity. J. Oral Microbiol. 10:1429788. doi: 10.1080/20002297.2018.1429788

Muras, A., Otero-Casal, P., Blanc, V., and Otero, A. (2020). Acyl homoserine lactone-mediated quorum sensing in the oral cavity: a paradigm revisited. Sci. Rep. 10:9800. doi: 10.1038/s41598-020-66704-4

Niu, C., Clemmer, K. M., Bonomo, R. A., and Rather, P. N. (2008). Isolation and characterization of an autoinducer synthase from Acinetobacter baumannii. J. Bacteriol. 190, 3386-3392. doi: 10.1128/JB.01929-07

Ochiai, S., Yasumoto, S., Morohoshi, T., and Ikeda, T. (2014). AmiE, a novel $N$-acylhomoserine lactone acylase belonging to the amidase family, from the activated-sludge isolate Acinetobacter sp. strain Ooi24. Appl. Environ. Microbiol. 80, 6919-6925. doi: 10.1128/AEM.02190-14

Oh, M. H., and Choi, C. H. (2015). Role of LuxIR homologue AnoIR in Acinetobacter nosocomialis and the effect of virstatin on the expression of anoR gene. J. Microbiol. Biotechnol. 25, 1390-1400. doi: 10.4014/jmb.1504.04069

Okshevsky, M., Regina, V. R., and Meyer, R. L. (2015). Extracellular DNA as a target for biofilm control. Curr. Opin. Biotechnol. 33, 73-80. doi: 10.1016/j.copbio. 2014.12.002

Peleg, A. Y., Jara, S., Monga, D., Eliopoulos, G. M., Moellering, R. C. Jr., and Mylonakis, E. (2009). Galleria mellonella as a model system to study Acinetobacter baumannii pathogenesis and therapeutics. Antimicrob. Agents Chemother. 53, 2605-2609. doi: 10.1128/AAC.01533-08

Peleg, A. Y., Seifert, H., and Paterson, D. L. (2008). Acinetobacter baumannii: emergence of a successful pathogen. Clin. Microbiol. Rev. 21, 538-582. doi: 10.1128/CMR.00058-07

Qi, L., Li, H., Zhang, C., Liang, B., Li, J., Wang, L., et al. (2016). Relationship between antibiotic resistance,biofilm formation, and biofilm-specific resistance in Acinetobacter baumannii. Front. Microbiol. 7:483. doi: 10.3389/fmicb.2016. 00483

Roca, I., Espinal, P., Vila-Farrés, X., and Vila, J. (2012). The Acinetobacter baumannii oxymoron: commensal hospital dweller turned pan-drug-resistant menace. Front. Microbiol. 3:148. doi: 10.3389/fmicb.2012.00148

Rumbo-Feal, S., Gómez, M. J., Gayoso, C., Álvarez-Fraga, L., Cabral, M. P., Aransay, A. M., et al. (2013). Whole transcriptome analysis of Acinetobacter 
baumannii assessed by RNA-sequencing reveals different mRNA expression profiles in biofilm compared to planktonic cells. PLoS One 8:e72968. doi: 10. 1371/journal.pone.0072968

Rumbo-Feal, S., Pérez, A., Ramelot, T. A., Álvarez-Fraga, L., Vallejo, J. A., Beceiro, A., et al. (2017). Contribution of the A. baumannii A1S_0114 gene to the interaction with eukaryotic cells and virulence. Front. Cell. Infect. Microbiol. 7:108. doi: 10.3389/fcimb.2017.00108

Sahu, P. K., Iyer, P. S., Oak, A. M., Pardesi, K. R., and Chopade, B. A. (2012). Characterization of eDNA from the clinical strain Acinetobacter baumannii AIIMS 7 and its role in biofilm formation. ScientificWorldJournal 2012:973436. doi: 10.1100/2012/973436

Saroj, S. D., and Rather, P. N. (2013). Streptomycin inhibits quorum sensing in Acinetobacter baumannii. Antimicrob. Agents Chemother. 57, 1926-1929. doi: 10.1128/AAC.02161-12

Shikuma, N. J., and Yildiz, F. H. (2009). Identification and characterization of OscR, a transcriptional regulator involved in osmolarity adaptation in Vibrio cholerae. J. Bacteriol. 191, 4082-4096. doi: 10.1128/JB.01540-08

Shin, B., Park, C., and Park, W. (2020). Stress responses linked to antimicrobial resistance in Acinetobacter species. Appl. Microbiol. Biotechnol. 104, 1423-1435. doi: 10.1007/s00253-019-10317-z

Smith, M. G., Gianoulis, T. A., Pukatzki, S., Mekalanos, J. J., Ornston, L. N., Gerstein, M., et al. (2007). New insights into Acinetobacter baumannii pathogenesis revealed by high-density pyrosequencing and transposon mutagenesis. Genes Dev. 21, 601-614.

Stacy, D. M., Le Quement, S. T., Hansen, C. L., Clausen, J. W., TokerNielsen, T., Brummond, J. W., et al. (2013). Synthesis and biological evaluation of triazole-containing $\mathrm{N}$-acyl homoserine lactones as quorum sensing modulators. Org. Biomol. Chem. 11, 938-954. doi: 10.1039/c2ob27 $155 \mathrm{a}$

Stacy, D. M., Welsh, M. A., Rather, P. N., and Blackwell, H. E. (2012). Attenuation of quorum sensing in the pathogen Acinetobacter baumannii using non-native $N$-acyl homoserine lactones. ACS Chem. Biol. 7, 1719-1728. doi: 10.1021/ cb300351x

Tetz, G. V., Artemenko, N. K., and Tetz, V. V. (2009). Effect of DNase and antibiotics on biofilm characteristics. Antimicrob. Agents Chemother. 53, 12041209. doi: 10.1128/AAC.00471-08

Tomaras, A. P., Dorsey, C. W., Edelmann, R. E., and Actis, L. A. (2003). Attachment to and biofilm formation on abiotic surfaces by Acinetobacter baumannii: involvement of a novel chaperone-usher pili assembly system. Microbiology 149, 3473-3484. doi: 10.1099/mic.0.26541-0

Tomaras, A. P., Flagler, M. J., Dorsey, C. W., Gaddy, J. A., and Actis, L. A. (2008). Characterization of a two-component regulatory system from Acinetobacter baumannii that controls biofilm formation and cellular morphology. Microbiology 154, 3398-3409. doi: 10.1099/mic.0.2008/019471-0

Towner, K. J. (2009). Acinetobacter: an old friend, but a new enemy. J. Hosp. Infect. 73, 355-363. doi: 10.1016/j.jhin.2009.03.032
Tucker, A. T., Nowicki, E. M., Boll, J. M., Knauf, G. A., Burdis, N. C., Trent, M. S., et al. (2014). Defining gene-phenotype relationships in Acinetobacter baumannii through one-step chromosomal gene inactivation. mBio 5:e131314. doi: 10.1128/mBio.01313-14

Vijayakumar, S., Rajenderan, S., Laishram, S., Anandan, S., Balaji, V., and Biswas, I. (2016). Biofilm formation and motility depend on the nature of the Acinetobacter baumannii clinical isolates. Front. Public Health 4:105. doi: 10. 3389/fpubh.2016.00105

Wood, C. R., Mack, L. E., and Actis, L. A. (2018a). An update on the Acinetobacter baumannii regulatory circuitry. Trends Microbiol. 26, 560-562. doi: 10.1016/j. tim.2018.05.005

Wood, C. R., Ohneck, E. J., Edelmann, R. E., and Actis, L. A. (2018b). A lightregulated type I pilus contributes to Acinetobacter baumannii biofilm, motility, and virulence functions. Infect Immun. 86:e0442-18. doi: 10.1128/IAI.004 42-18

Yakkala, H., Samantarrai, D., and Gribskov, M. (2019). Comparative genome analysis reveals niche-specific genome expansion in Acinetobacter baumannii strains. PLoS One 14:e0218204. doi: 10.1371/journal.pone.0218204

Yang, C. H., Su, P. W., Moi, S. H., and Chuang, L. Y. (2019). Biofilm formation in Acinetobacter baumannii: genotype-phenotype correlation. Molecules 24:1849. doi: 10.3390/molecules24101849

Zeighami, H., Valadkhani, F., Shapouri, R., Samadi, E., and Haghi, F. (2019). Virulence characteristics of multidrug resistant biofilm forming Acinetobacter baumannii isolated from intensive care unit patients. BMC Infect. Dis. 19:629. doi: 10.1186/s12879-019-4272-0

Zhang, Y., Brackman, G., and Coenye, T. (2017). Pitfalls associated with evaluating enzymatic quorum quenching activity: the case of MomL and its effect on Pseudomonas aeruginosa and Acinetobacter. PeerJ 5:e3251. doi: 10.7717/peerj. 3251

Conflict of Interest: The enzyme Aii20J, used in some experiments described in this work, is protected by the following patent: AO, MR, and CM (2016). Peptide with quorum-sensing inhibitory activity, polynucleotide that encodes said peptide, and the uses thereof. PCT/ES2014/070569.

The remaining authors declare that the research was conducted in the absence of any commercial or financial relationships that could be construed as a potential conflict of interest.

Copyright (c) 2020 Mayer, Muras, Parga, Romero, Rumbo-Feal, Poza, Ramos-Vivas and Otero. This is an open-access article distributed under the terms of the Creative Commons Attribution License (CC BY). The use, distribution or reproduction in other forums is permitted, provided the original author(s) and the copyright owner(s) are credited and that the original publication in this journal is cited, in accordance with accepted academic practice. No use, distribution or reproduction is permitted which does not comply with these terms. 